hep-th/0406147

\title{
The Complex Geometry of Holographic Flows of Quiver Gauge Theories
}

\author{
Nick Halmagyi ${ }^{(1)}$, Krzysztof Pilch ${ }^{(2),(1) \dagger}$, \\ Christian Römelsberger ${ }^{(1)}$ and Nicholas P. Warner ${ }^{(1)}$ \\ (1) Department of Physics and Astronomy \\ University of Southern California \\ Los Angeles, CA 90089-0484, USA \\ (2) Departament ECM, Facultat Física \\ Universitat de Barcelona \\ Diagonal 647 \\ 08028 Barcelona, Spain
}

\begin{abstract}
We argue that the complete Klebanov-Witten flow solution must be described by a Calabi-Yau metric on the conifold, interpolating between the orbifold at infinity and the cone over $T^{(1,1)}$ in the interior. We show that the complete flow solution is characterized completely by a single, simple, quasi-linear, second order PDE, or "master equation," in two variables. We show that the Pilch-Warner flow solution is almost Calabi-Yau: It has a complex structure, a hermitian metric, and a holomorphic (3,0)-form that is a square root of the volume form. It is, however, not Kähler. We discuss the relationship between the master equation derived here for Calabi-Yau geometries and such equations encountered elsewhere and that govern supersymmetric backgrounds with multiple, independent fluxes.
\end{abstract}

June 2004

$\dagger$ On sabbatical leave. 


\section{Introduction}

One of the first families of physical, supersymmetric holographic flows to be studied were those [1.2] describing the massive, $\mathcal{N}=1$ supersymmetric flows from $\mathcal{N}=2$ quiver gauge theories. These flows were shown to go to Leigh-Strassler type fixed point theories in the infra-red, and the holographic duals of these fixed points were also identified. It has remained one of the unsolved problems in holography to construct these solutions explicitly, and while this is hard for the general quiver theories, one might expect to be

able to solve this problem for the $\widehat{A}_{1}$ quiver of [1] because of its much higher level of symmetry. Despite numerous attempts, there has been rather little progress in finding the flow solution within supergravity.

Shortly after the work of [1,:2], new supergravity fixed points were discovered in [3], and the corresponding flow solutions were obtained in [4]. While these flows were originally investigated for $\mathcal{N}=4$ Yang-Mills theory, it was noted at the time that these solutions were also flows in the untwisted sector of the $\mathcal{N}=2$ superconformal quiver gauge theories obtained via orbifolds. These supergravity solutions were originally constructed in five dimensions, but their complete ten-dimensional analogs were subsequently constructed in [5,6], and then significantly generalized in a recent paper [7]. While these supergravity solutions were explicitly known, the problem was that their underlying geometric structure was far from evident: The solutions were obtained via the magic of consistent truncation, and not through a clearer geometric principle. The complication is that these solutions involve multiple, non-trivial fluxes and are not some straightforward decoration of a CalabiYau geometry.

Finally, in [8] it was argued that the holographic flow solutions of [1],2] and [3, t] should be related via a continuous family of duality transformations. Further evidence for this was found in the field theory analysis of [9].

Our purpose in this paper is to re-examine and understand the geometry of both of these families of flows using, in particular, the ideas of [10,11, [, 12] for classifying the "supersymmetry bundles." For the Klebanov-Witten flow we will argue that the internal geometry of the entire flow solution must live on the singular conifold. That is, the internal manifold must be governed by a Ricci-flat, Kähler metric on the conifold, and that this metric must interpolate between the orbifold, $\mathbb{C}^{2} / \mathbb{Z}_{2} \times \mathbb{C}$ at infinity and the cone over $T^{(1,1)}$ in the interior. The orbifold singularity at infinity is thus blown up as one moves radially inwards. We then proceed to characterize this geometry exactly in terms 
of a "master equation" that is a single, second-order, quasi-linear PDE in two variables. This characterization of the solution represents a significant simplification over the more traditional Monge-Ampère equation (which is strongly non-linear, and sixth-order).

For the Pilch-Warner (PW) flow we re-examine the geometry underlying the general class of solutions in [7]. We will show that the internal manifold has an integrable complex structure and a holomorphic $(3,0)$-from such that $\Omega \wedge \bar{\Omega}$ is the volume form of the manifold. The internal geometry is thus almost Calabi-Yau, except that it is not Kähler: The Kähler form is not closed. It was also shown in [7] that this non-trivial flux geometry was governed by a single, second-order, quasi-linear PDE, or "master equation." Indeed, this master equation is very simply related to the one that we find here for the Calabi-Yau geometry of the Klebanov-Witten flow.

This observation now provides new insight into the broader class of results contained in [10,11, [1, 12]. These were all supersymmetric, multiple flux solutions, and were all governed by a single master equation similar to those encountered here. Our work here suggests that all these multiple flux solutions are indeed some relatively simple deformation of a Calabi-Yau condition [13].

In section 2 we will use a combination of field theory, supergravity and brane-probe analysis to argue that the complete Klebanov-Witten flow should be described by a CalabiYau metric on the singular conifold. In section 3 we look at the conifold geometry in some detail, and use the symmetries of the flow that we seek to pin down the general form of the metric on the internal space. Having obtained a suitably general metric Ansatz, in section 4 we use the techniques of [10, 11, 7, 12] to solve the Killing spinor equations, and thereby obtain the Calabi-Yau metric in terms of the solution of the master equation. In section 5 we repeat the analysis, but this time more systematically in terms of the special holonomy of the spin connection. The end-result is the same as that of section 4 , but one sees very explicitly how each equation emerges from distinct conditions on the holonomy. Section 5 also contains a more detailed discussion of the complex structure and coordinates, and contrasts our approach with adaptions of the ideas of Tian and Yau, which would lead to the Monge-Ampère equation. Section 6 contains our geometric re-examination of the geometry of the PW flow, and section 7 contains some final remarks. 


\section{Field Theory}

The UV point of the Klebanov-Witten flow is the $\mathcal{N}=2, \widehat{A}_{1}$ quiver gauge theory. This has gauge group $S U(N) \times S U(N)$ and also has two bi-fundamental hypermultiplets. In terms of $\mathcal{N}=1$ multiplets, the two $\mathcal{N}=2$ vector multiplets contain two $\mathcal{N}=1$ adjoint chiral superfields $\Phi_{1}, \Phi_{2}$ while each of the hypermulitplets yields a pair of chiral multiplets $\left(A_{1}, B_{1}\right)$ and $\left(B_{2}, A_{2}\right)$. The chiral superfields $A_{1}, B_{2}$ lie in the $(N, \bar{N})$ representation while $B_{1}, A_{2}$ are in the $(\bar{N}, N)$. The superpotential is

$$
\begin{aligned}
W & =\operatorname{Tr}\left[\left(\begin{array}{cc}
\Phi_{1} & 0 \\
0 & \Phi_{2}
\end{array}\right)\left[\left(\begin{array}{cc}
0 & A_{1} \\
A_{2} & 0
\end{array}\right),\left(\begin{array}{cc}
0 & B_{2} \\
B_{1} & 0
\end{array}\right)\right]\right] \\
& =\operatorname{Tr}\left[\Phi_{1}\left(A_{1} B_{1}-B_{2} A_{2}\right)\right]+\operatorname{Tr}\left[\Phi_{2}\left(A_{2} B_{2}-B_{1} A_{1}\right)\right] .
\end{aligned}
$$

This theory has a $S U(2) \times U(1) \mathcal{R}$-symmetry together with a global $S U(2)$ symmetry under which the two hypermultiplets form a doublet.

The Klebanov-Witten flow is driven by the relevant operator

$$
\Delta W=\frac{1}{2} m \operatorname{Tr}\left[\Phi_{1}^{2}\right]-\frac{1}{2} m \operatorname{Tr}\left[\Phi_{2}^{2}\right]
$$

which breaks the $\mathcal{N}=2$ supersymmetry of the vector multiplet. The IR point of this flow has only the bi-fundamental fields, the adjoint fields having been integrated out. It has a superpotential

$$
W_{\mathcal{N}=1}=\lambda \operatorname{Tr}\left(A_{1} B_{1} B_{2} A_{2}-A_{1} A_{2} B_{2} B_{1}\right) .
$$

The methods of Leigh and Strassler [14] suggest that this is a non-trivial CFT [1].

This theory still has the $S U(2)$ global symmetry but the $S U(2) \times U(1) \mathcal{R}$-symmetry is broken to a $U(1) \mathcal{R}$-symmetry. There are also two $\mathbb{Z}_{2} \mathcal{R}$-symmetries. One of them is the symmetry exchanging the two gauge groups, while the other symmetry is charge conjugation [1]. The obvious interchange symmetry is:

$$
A_{1} \leftrightarrow A_{2}, \quad B_{1} \leftrightarrow B_{2}, \quad \Phi_{1} \leftrightarrow \Phi_{2} .
$$

However, this is not a symmetry of the perturbation (2.2). To get a symmetry of the complete perturbed superpotential we need to combine the interchange with an $\mathcal{R}$-symmetry transformation:

$$
A_{1} \rightarrow i A_{2}, \quad A_{2} \rightarrow i A_{1}, \quad B_{1} \rightarrow i B_{2}, \quad B_{2} \rightarrow i B_{1}, \quad \Phi_{1} \leftrightarrow \Phi_{2},
$$

under which $W+\Delta W \rightarrow-(W+\Delta W)$, and hence the action is invariant. We will need this particular symmetry later. 


\subsection{The brane-probe theory}

A fundamental feature of D-brane physics is that since they are BPS objects, D-branes are free to move in their tranverse directions without feeling a force from parallel branes. This manifests itself as a moduli space in the gauge theory living on the branes. In this section we use this Higgs moduli space along the Klebanov-Witten flow to obtain important insights into the geometry of the holographic dual theory.

When a single brane moves off the stack and probes the internal geometry, the gauge group is broken according to:

$$
S U(N+1) \times S U(N+1) \rightarrow S U(N) \times U(1) \times S U(N) \times U(1) .
$$

Since $N \gg 1$, the $S U(N)$ factors produce the background whereas the $U(1)$ factors correspond to the brane-probe theory, and their gravitational effects can be neglected. If the probe brane is located far away from the rest of the branes, the $\mathrm{W}$ bosons are very heavy and the $U(1)$ part of the theory decouples. We now concentrate on the decoupled $U(1) \times U(1)$ gauge theory. The diagonal $U(1)$ factor is just a free $U(1)$ and we will drop it.

The anti diagonal $U(1)$ couples to four scalars $A_{1}, A_{2}, B_{1}$ and $B_{2}$. The fields $A_{1}$ and $B_{2}$ have a $U(1)$ charge +1 , wheras $A_{2}$ and $B_{1}$ have charge -1 . Since these matter fields have large vevs, the remaining $U(1)$ can be integrated out. This corresponds to a Kähler quotient. Alternatively, the Kähler quotient is equivalent to solving D-flatness modulo this $U(1)$.

The resulting space of vacua is parameterized by the gauge invariant variables

$$
z_{1}=A_{1} B_{1}, \quad z_{2}=B_{1} B_{2}, \quad z_{3}=A_{2} A_{1} \quad \text { and } \quad z_{4}=B_{2} A_{2}
$$

These variables satisfy

$$
z_{1} z_{4}-z_{2} z_{3}=0
$$

the defining equation of the singular conifold.

This result was deduced from the structure of the $F$-terms of the field theory, and one should note that, provided that there are no non-perturbative corrections, this analysis is valid not only valid for the IR fixed point, but for all points along the RG flow. As a result, the moduli space of the brane probes for the complete flow should be described by the conifold, and in particular it should inherit its complex structure. Since the $D$-terms 
of the field theory action undergo strong renormalization, the metric of the field theory, and of the brane probes will be non-trivial. We therefore infer that as far as the brane probes are concerned, the flow will be described by some hermitian metric on the conifold.

To get a better picture of what is happening in the UV, it is convenient to change coordinates to:

$$
w_{1}=z_{1}+z_{4}, \quad w_{2}=i\left(z_{2}+z_{3}\right), \quad w_{3}=z_{2}-z_{3} \quad \text { and } \quad w_{4}=i\left(z_{1}-z_{4}\right)
$$

which satisfy:

$$
w_{1}^{2}+w_{2}^{2}+w_{3}^{2}+w_{4}^{2}=0 .
$$

Solving the F-flatness condition in (2.1) and (2.2) for the $\Phi_{i}$ yields

$$
m \Phi_{1}=-\left(A_{1} B_{1}-B_{2} A_{2}\right), \quad m \Phi_{2}=A_{2} B_{2}-B_{1} A_{1}
$$

and since the probe theory is Abelian, we can write this as

$$
w_{4}=i m \Phi_{1}=i m \Phi_{2}
$$

Thus the moduli space has a preferred coordinate, $w_{4}$, that is dual to the vev of the scalar fields in $\mathcal{N}=2$ vector multiplet. Note that at the UV fixed point, when $m=0$, one has $w_{4}=0$ and $\Phi_{1}+\Phi_{2}$ is a freely choosable, independent field. This means that in the UV the probe moduli space is simply the orbifold, $\mathbb{C}^{2} / \mathbb{Z}_{2} \times \mathbb{C}$, and that the conifold has degenerated. At the UV point, the scalar kinetic term and the brane probe metric have the trivial Euclidean, flat form while in the IR the brane-probe moduli space lies on the singular conifold with the Calabi-Yau metric obtained from the cone over $T^{(1,1)}$ [1]. This naturally leads one to consider the family of manifolds

$$
w_{1}^{2}+w_{2}^{2}+w_{3}^{2}+\epsilon^{2} w_{4}^{2}=0
$$

For an arbitrary, non-zero value of $\epsilon$ we can rescale $w_{4}$ and reproduce (2.10), however the Kähler metric on the moduli space will make it possible to determine the size of the constant $\epsilon$. Thus by varying $\epsilon$ between 0 and 1 we can interpolate between $\mathbb{C}^{2} / \mathbb{Z}_{2} \times \mathbb{C}$ and the singular conifold.

Whilst the previous calculation is not new or difficult, our interpretation of the result is our guide to the construction of the dual geometry of the whole flow. Namely, it suggests that the holographic dual of the entire flow has the complex structure of the 
conifold. However the Kähler metric on this conifold has to be squeezed so as to ultimately degenerate to the orbifold in the UV. To obtain the exact result we need to determine a squeezed Kähler metric on the conifold that respects the symmetries of the flow. One might hope to get this metric from the field theory, however quantum corrections mean that the metric on the brane-probe moduli space doesn't necessarily coincide with the metric obtained from the Kähler quotient of the field theory metric obtained from the UV limit [15].

\subsection{Supergravity}

Having gained some insight from the field theory, we now turn more directly to the gravity side of the duality. In addition to the dual geometry we must consider the possibility of non-trivial profiles for the fluxes and the dilaton of IIB supergravity.

The $\mathcal{N}=2$ quiver gauge theory can be realized in string theory by D3-branes probing a $\mathbb{C}^{2} / \mathbb{Z}_{2}$ orbifold singularity [16]. The mass deformation (2.2) is then described by a twisted-sector, closed string mode. The corresponding twisted-sector, closed string modes of type IIB theory on an ALE orbifold come in six-dimensional $\mathcal{N}=(2,0)$ tensor multiplets associated to the blow-up of a two-cycle. Each such multiplet contains five scalars: Three of these scalars are hyper-Kähler deformations while the remaining two scalars describe the NS and RR two-form flux through the two-cycle. Putting D3-branes on the singularity breaks supersymmetry to four-dimensional $\mathcal{N}=2$ supersymmetry. The two scalars describing the two-form flux then couple at first order to the relative gauge couplings and theta angles of the gauge theory. On the other hand the three scalars describing the hyperKähler blow-up modes couple to the D-terms and F-terms [8]. This shows, that at leading order our mass deformation corresponds to a geometric deformation (2.13) of the orbifold and that the two-form potentials and three-form fluxes are not being turned on. Also, to leading order the dilaton-axion is not running. We will therefore seek supergravity with no such fluxes, and with a trivial dilaton and axion.

The brane-probe analysis also yields more evidence for this proposal. If the holographic dual theory had non-zero three-form flux, then one would expect there to be a potential for the brane-probe that would restrict it to a subspace of less than six dimensions [17]. Since we found a six-dimensional brane-probe moduli space, it seems natural that there should be no three-form fluxes. 
We can now use the fact that our D3-branes can probe the whole geometry. In order for the probe brane to preserve supersymmetry, the Killing spinor, $\epsilon$, in the holographic dual theory has to satisfy [18,19]:

$$
\Gamma^{1234} \epsilon= \pm i \epsilon
$$

(for one uniform choice of sign, depending upon conventions) at the location of the probe brane. Since the probe brane can be moved anywhere in the geometry, equation (2.14) has to hold at all points. We can utilise results from [20] where it was established that such solutions are of Becker-type.

This means that the internal manifold is Calabi-Yau, in agreement with our conclusions from the previous section. In Becker type compactifications the warp factor in front of the metric parallel to the branes is $Z^{-\frac{1}{2}}$, where $Z$ satisfies a harmonic equation, and the five-form flux is related to the warp factor by:

$$
f=-\frac{1}{4 Z}
$$

where the five-form flux is given by

$$
F=(1+*) d f \wedge d x^{1} \wedge d x^{2} \wedge d x^{3} \wedge d x^{4}
$$

There could be a holomorphic dilaton or imaginary self dual three-form fluxes, but we already agued that they vanish.

The fact that the internal geometry is Calabi-Yau implies the that the Killing spinors can be determined using three independent helicity projectors of the form:

$$
\Pi^{(a b)}=\frac{1}{2}\left(\mathbb{1}-i \Gamma^{a} \Gamma^{b}\right)
$$

for some frame indices, $a$ and $b$, on the Calabi-Yau manifold. These helicity projectors have the effect of isolating the spinor that is a singlet under the $S U(3)$ holonomy.

In summary, we are searching for a Calabi-Yau metric on the singular conifold with an $S U(2) \times U(1)$ symmetry, and the discrete, $\mathbb{Z}_{2} \mathcal{R}$ symmetries described in [1]. The metric must agree with the more standard metric [21] for small values of the radial coordinate, and it must reduce to a flat metric on $\mathbb{C}^{2} / \mathbb{Z}_{2} \times \mathbb{C}$ in limit of some large appropriately chosen radial coordinate. 


\section{The conifold, its complex structure and hermitian metrics}

\subsection{Describing the conifold}

As explained in [21], it is very convenient to describe the conifold in terms of the matrix:

$$
\mathcal{W} \equiv \frac{1}{\sqrt{2}}\left(\begin{array}{cc}
w_{3}+i w_{4} & w_{1}-i w_{2} \\
w_{1}+i w_{2} & -w_{3}+i w_{4}
\end{array}\right)
$$

The vanishing of the determinant of this matrix defines the conifold:

$$
\operatorname{det}(\mathcal{W})=-\frac{1}{2}\left(w_{1}^{2}+w_{2}^{2}+w_{3}^{2}+w_{4}^{2}\right)=0
$$

while the obvious norm defines a natural radial coordinate:

$$
\operatorname{Tr}\left(\mathcal{W}^{\dagger} \mathcal{W}\right)=\left|w_{1}\right|^{2}+\left|w_{2}\right|^{2}+\left|w_{3}\right|^{2}+\left|w_{4}\right|^{2} \equiv r^{2}
$$

The surface of constant $r$ is the $T^{(1,1)}$ space:

$$
T^{(1,1)}=\frac{S U(2) \times S U(2)}{U(1)},
$$

which can be given a Einstein metric [22]. Taking the cone over this leads to the wellknown Ricci-flat metric on the conifold [21,1]. This metric has $S U(2)_{L} \times S U(2)_{R} \times U(1)$ invariance where the $S U(2)$ 's act on the left and right of $\mathcal{W}$, while the $U(1)$ is an overall phase rotation on the $w_{j}$.

As described in the previous section, we seek a broader class of metrics on the conifold: Metrics that preserve the complex structure and have an $S U(2) \times U(1)$ symmetry where the $U(1)$ is the phase rotation on the $w_{j}$ and the $S U(2)$ is the diagonal subgroup of $S U(2)_{L} \times S U(2)_{R}$. Note that this particular $S U(2)$ leaves $w_{4}$ invariant, while transforming $\left(w_{1}, w_{2}, w_{3}\right)$ as a triplet. Thus the continuous symmetry acts as $S O(3) \times U(1)$.

Also note that if one uses the identification of coordinates and fields in (2.7) and (2.9) then the discrete symmetry, (2.5), of the field theory becomes the reflection:

$$
w_{1} \rightarrow-w_{1}, \quad w_{2} \rightarrow-w_{2}, \quad w_{3} \rightarrow-w_{3}, \quad w_{4} \rightarrow w_{4} .
$$

Thus we are actually looking for an $O(3) \times U(1)$ invariant metric. 


\subsection{Breaking some symmetry and a degenerate limit}

Consider the "trivial" deformation of the conifold:

$$
w_{1}^{2}+w_{2}^{2}+w_{3}^{2}+\varepsilon^{2} w_{4}^{2}=0 .
$$

As we discussed earlier, the parameter, $\varepsilon$, can be removed by rescaling $w_{4}$. However, once one has chosen a metric on the conifold, this scale parameter has meaning, and breaks the symmetry to $O(3) \times U(1)$. Moreover, if one takes the degenerate limit $\varepsilon=0$ this modifies the complex structure and enhances the symmetry to $S U(2) \times U(1) \times U(1)$. In particular, the underlying complex manifold becomes the orbifold $\mathbb{C}^{2} / \mathbb{Z}_{2} \times \mathbb{C}$. The single factor of $\mathbb{C}$ is simply parametrized by $w_{4}$, while the $\mathbb{C}^{2}$ can be parametrized by two coordinates $\left(\zeta_{1}, \zeta_{2}\right)$ that are identified under $\zeta_{j} \rightarrow-\zeta_{j}$. The invarants under this action are:

$$
w_{1}=\frac{1}{2}\left(\zeta_{1}^{2}+\zeta_{2}^{2}\right), \quad w_{2}=\frac{i}{2}\left(\zeta_{1}^{2}-\zeta_{2}^{2}\right), \quad w_{3}=i \zeta_{1} \zeta_{2}
$$

and these satisfy (3.6) with $\varepsilon=0$. Note that under the residual $S U(2)$ symmetry $\left(\zeta_{1}, \zeta_{2}\right)$ transforms as a doublet. The two $U(1)$ symmetries are the now-independent phase rotations of $\left(\zeta_{1}, \zeta_{2}\right)$ and $w_{4}$.

Note that in this degeneration limit we have recovered the underlying manifold and symmetry structure of the $\mathbb{Z}_{2}$ orbifold of the solutions considered in [5].6]. We will discuss this further in section 6 .

\subsection{Some useful coordinates}

We wish to parametrize the conifold in terms of the residual $S U(2) \times U(1)$ symmetry. To this end, introduce:

$$
\mathcal{W}_{0} \equiv \frac{1}{\sqrt{2}} \sqrt{\mu^{2}-\nu^{2}} \mathbb{1}+\frac{1}{\sqrt{2}}\left(\begin{array}{cc}
0 & \mu+\nu \\
\mu-\nu & 0
\end{array}\right)
$$

where $\mu, \nu$ and $\phi$ are real, with $|\mu| \geq|\nu|$. A general matrix of the form (3.1) can then be obtained by conjugating with a single matrix, $\mathcal{L} \in S U(2)$, and multiplying by a phase, $e^{i \phi} \in U(1):$

$$
\mathcal{W}=e^{i \phi} \mathcal{L} \mathcal{W}_{0} \mathcal{L}^{\dagger}
$$

One can see that this is possible by considering the real and imaginary parts of $w_{j}=$ $x_{j}+i y_{j}$, and denoting the first three components by 3 -vectors $\vec{x}$ and $\vec{y}$. First use the overall phase to set $x_{4}=0$, and then the imaginary part of the conifold equation (3.2) 
means that $\vec{x} \cdot \vec{y}=0$. One can now use all of the $S O(3)$ symmetry to put these orthogonal vectors in the form $\vec{x}=(\mu, 0,0)$ and $\vec{y}=(0, \nu, 0)$ for some $\mu, \nu>0$. The real part of the conifold equation implies $\mu^{2}=\nu^{2}+y_{4}^{2}$, and hence $\mu \geq \nu$. We thus obtain $w_{1}=\mu, w_{2}=i \nu$, $w_{3}=0$ and $w_{4}=i \sqrt{\mu^{2}-\nu^{2}}$, which is (3.8), provided that we let $\mu$ and $\nu$ be both positive or negative with $|\mu| \geq|\nu|$.

We parametrize the $S U(2)$ matrices in terms of Euler angles. That is, we write

$$
\mathcal{L}=\mathcal{R}_{12}\left(\varphi_{2}\right) \mathcal{R}_{13}\left(\varphi_{1}\right) \mathcal{R}_{12}\left(\varphi_{3}\right)
$$

where

$$
\mathcal{R}_{12}(\varphi) \equiv\left(\begin{array}{cc}
e^{-\frac{i}{2} \varphi} & 0 \\
0 & e^{+\frac{i}{2} \varphi}
\end{array}\right), \quad \mathcal{R}_{13}(\varphi) \equiv\left(\begin{array}{cc}
\cos \left(\frac{1}{2} \varphi\right) & -\sin \left(\frac{1}{2} \varphi\right) \\
\sin \left(\frac{1}{2} \varphi\right) & \cos \left(\frac{1}{2} \varphi\right)
\end{array}\right) .
$$

It is convenient to introduce the invariant 1 -forms, $\sigma_{j}$ where:

$$
\mathcal{L}^{\dagger} d \mathcal{L}=\frac{1}{2}\left(\begin{array}{cc}
i \sigma_{3} & \sigma_{1}-i \sigma_{2} \\
-\left(\sigma_{1}+i \sigma_{2}\right) & -i \sigma_{3}
\end{array}\right)
$$

Explicitly, one has:

$$
\begin{aligned}
\sigma_{1} & \equiv \cos \varphi_{3} d \varphi_{1}+\sin \varphi_{3} \sin \varphi_{1} d \varphi_{2}, \\
\sigma_{2} & \equiv \sin \varphi_{3} d \varphi_{1}-\cos \varphi_{3} \sin \varphi_{1} d \varphi_{2}, \\
\sigma_{3} & \equiv \cos \varphi_{1} d \varphi_{2}+d \varphi_{3} .
\end{aligned}
$$

For future reference we note that in these coordinates, the degenerate limit that takes us from the conifold to the orbifold $\mathbb{C}^{2} / \mathbb{Z}_{2} \times \mathbb{C}$ is to take $|\mu|,|\nu| \rightarrow \infty$ with $\mu-\nu$ finite, or $|\mu|,|\nu| \rightarrow \infty$ with $\mu+\nu$ finite.

\subsection{The $(1,0)$-forms}

Since the conifold is a 3 -fold, there are three independent $(1,0)$-forms at each point, and it turns out there there is a $S U(2) \times U(1)$ invariant basis for these differentials. In terms of complex coordinates, these are $\sum_{j=1}^{3} \bar{z}_{j} d z_{j}, \bar{z}_{4} d z_{4}$ and $\bar{z}_{4}\left(\epsilon^{i j k} \bar{z}_{i} z_{j} d z_{k}\right)$, while in terms of the coordinates introduced above, these may be reduced to the equivalent basis:

$$
\begin{aligned}
& \omega_{1} \equiv d \mu+i\left(\mu d \phi+\nu \sigma_{3}\right), \\
& \omega_{2} \equiv d \nu+i\left(\nu d \phi+\mu \sigma_{3}\right) \\
& \omega_{3} \equiv \mu \sigma_{1}+i \nu \sigma_{2} .
\end{aligned}
$$

It is instructive to note that the degenerate conifold also has the $S U(2)$ invariant (1,0)-form:

$$
\omega_{0} \equiv \zeta_{2} d \zeta_{1}-\zeta_{1} d \zeta_{2} \sim\left(\sigma_{1}+i \sigma_{2}\right)=\frac{1}{2 u v}\left((\mu+\nu) \omega_{3}+(\mu-\nu) \bar{\omega}_{3}\right)
$$

Observe that while this is a $(1,0)$-form in the complex structure of the degenerate conifold, it is not of type $(1,0)$ in the complex structure of the non-degenerate conifold. Thus the limit $\varepsilon \rightarrow 0$ in (3.6) involves a discontinuity in the complex structure. 


\subsection{Hermitian metrics}

The most general hermitian metric on the conifold can be written in the form

$$
d s^{2}=g_{i \bar{\jmath}} \omega^{i} \bar{\omega}^{\bar{\jmath}}
$$

for some metric coefficients, $g_{i \bar{\jmath}}$, that are arbitrary functions of the coordinates. The $S U(2) \times S U(2) \times U(1)$ invariant, Ricci-flat metric on the conifold is:

$$
\begin{aligned}
d s^{2} & =\frac{1}{3} u^{-\frac{2}{3}}\left(\frac{1}{3}\left|\omega_{1}\right|^{2}+\left|\omega_{2}\right|^{2}+\left|\omega_{3}\right|^{2}+\frac{1}{\left(\mu^{2}-\nu^{2}\right)}\left|\mu \omega_{1}-\nu \omega_{2}\right|^{2}\right) \\
& =d \rho^{2}+\frac{1}{3} \rho^{2}\left(d \theta^{2}+\sigma_{1}^{2}+\cos ^{2} \theta \sigma_{2}^{2}+\sin ^{2} \theta \sigma_{3}^{2}+\frac{4}{3}\left(d \phi+\cos \theta \sigma_{3}\right)^{2}\right) .
\end{aligned}
$$

where $\mu=\rho^{3 / 2}$ and $\nu=\rho^{3 / 2} \cos \theta$. This is the cone over $T^{(1,1)}$, but parametrized in a slightly unusual manner. Remember that we want to preserve and make manifest the diagonal $S U(2)$ in $S U(2)_{L} \times S U(2)_{R}$. If one does this on the $S^{2} \times S^{2}$ base of the $T^{(1,1)}$ space then one can write the standard metric on this product space as:

$$
\begin{aligned}
d s_{4}^{2} & =\left(d \theta_{1}^{2}+\sin ^{2} \theta_{1} d \phi_{1}^{2}\right)+\left(d \theta_{2}^{2}+\sin ^{2} \theta_{2} d \phi_{2}^{2}\right) \\
& =2\left(d \theta^{2}+\sigma_{1}^{2}+\cos ^{2} \theta \sigma_{2}^{2}+\sin ^{2} \theta \sigma_{3}^{2}\right) .
\end{aligned}
$$

Since $d\left(2 \cos \theta \sigma_{3}\right)$ yields a Kähler form on (3.18), we see that (3.17) involves the proper Hopf fibration to make $T^{(1,1)}$.

Imposing the condition that the metric be $S O(3) \times U(1)$ invariant implies that the metric can only depend explicitly upon $\mu$ and $\nu$. It is also easy to see that the $\mathbb{Z}_{2}$ symmetry:

$$
\varphi_{3} \rightarrow \varphi_{3}+\pi \quad \Rightarrow \quad \sigma_{1} \rightarrow-\sigma_{1}, \quad \sigma_{2} \rightarrow-\sigma_{2}
$$

is exactly that of (3.5). This is because $w_{4}=-i \sqrt{\mu^{2}-\nu^{2}} e^{i \phi}$ is invariant under the shift of $\varphi_{3}$, while in the conjugation by $\mathcal{L}$, this shift has the effect of negating all the entries in the second matrix of (3.8). (In section 5 we will give explicit formulae for the $w_{j}$, from which this result will be obvious.)

Requiring that the metric be invariant under this $\mathbb{Z}_{2}$ symmetry has the effect of requiring that $\omega_{3}$ only appear paired with $\bar{\omega}_{3}$ in (3.16). The metric thus has the form:

$$
d s^{2}=Q_{1} d \mu^{2}+Q_{2} d \nu^{2}+Q_{3} d \mu d \nu+Q_{4}\left|\omega_{3}\right|^{2}+Q_{5}\left(\sigma_{3}+Q_{6} d \phi\right)^{2}+Q_{7} d \phi^{2},
$$

for some arbitrary functions, $Q_{j}(\mu, \nu)$. By introducing a new coordinate, $u(\mu, \nu)$, we can convert the $(\mu, \phi)$ part of this metric to $\hat{Q}_{1}\left(d u^{2}+u^{2} d \phi^{2}\right)$, for some new function $\hat{Q}_{1}$. By 
introducing a new coordinate, $v(u, \nu)$, we can then eliminate the $d u d \nu$ term. This then leads to our final metric Ansatz:

$$
d s^{2}=H_{1}^{2}\left(d u^{2}+u^{2} d \phi^{2}\right)+H_{2}^{2} d v^{2}+H_{3}^{2} \sigma_{1}^{2}+H_{4}^{2} \sigma_{2}^{2}+H_{5}^{2} v^{2}\left(\sigma_{3}+H_{6} d \phi\right)^{2}
$$

for some arbitrary functions, $H_{j}(u, v)$. The existence of the complex structure with respect to which the metric (3.21) is hermitian imposes relations upon the $H_{j}$, but we will not make this assumption, and instead recover it from the supersymmetry variations. Our starting point will therefore be this metric Ansatz. However we should mention, that a $u$-independent re-definition of $v$ does not change the form of this Ansatz. We will later see how to choose a good gauge for this.

Note that this metric Ansatz is a simple generalization of the one employed in [7].

\section{The Calabi-Yau metric from the Killing spinors}

The traditional way to obtain a "simple" equation the Ricci-flat metric on the a Kähler manifold is to write down the Ricci-flat condition in terms of the Kähler potential. This generates a Monge-Ampère equation, which, for a 3-fold is a sixth-order, non-linear PDE. There are existence theorems for solutions of this equation [23,24], and these will be discussed later.

The problem we wish to solve here has some extra global symmetry, and we will use the supersymmetry in an approach that closely follows that of [10,11, 1, 12]. That is, we start with the metric Ansatz (3.21), make an algebraic prescription for the supersymmetry bundle, and go on to derive all the essential equations from the supersymmetry variations. The result is significantly simpler than the direct output of the Monge-Ampère equation.

\subsection{Solving the Ansatz}

We begin by introducing the frames:

$$
\begin{aligned}
& e^{1} \equiv H_{1} d u, \quad e^{2} \equiv H_{2} d v, \quad e^{3} \equiv H_{3} \sigma_{1}, \quad e^{4} \equiv H_{4} \sigma_{2}, \\
& e^{5} \equiv H_{5} v\left(\sigma_{3}+H_{6} d \phi\right), \quad e^{6} \equiv H_{1} u d \phi
\end{aligned}
$$

This metric has a natural almost-complex structure (indeed, it is the one inherited from the conifold, and will ultimately become a Kähler form):

$$
J=e^{1} \wedge e^{6}+e^{3} \wedge e^{4}+e^{2} \wedge e^{5} .
$$


Based upon this, we introduce the helicity projectors:

$$
\Pi_{1}=\frac{1}{2}\left(\mathbb{1}-i \Gamma^{1} \Gamma^{6}\right), \quad \Pi_{2}=\frac{1}{2}\left(\mathbb{1}-i \Gamma^{3} \Gamma^{4}\right), \quad \Pi_{3}=\frac{1}{2}\left(\mathbb{1}-i \Gamma^{2} \Gamma^{5}\right),
$$

and look for covariant constant spinors that satisfy:

$$
\Pi_{j} \epsilon=0, \quad j=1,2,3 .
$$

There will then be a second covariant constant spinor that can be obtained by complex conjugation and whose helicities are thus exactly the opposite of those in (4.3), (4.4).

In order to solve

$$
\nabla_{\mu} \epsilon=0
$$

it is helps to fix the dependence on the coordinates as far as possible before analyzing the equations in detail. The dependence upon angular coordinates can be obtained using the symmetries and the Lie derivative on spinors

$$
L_{K} \epsilon \equiv K^{\mu} \nabla_{\mu} \epsilon+\frac{1}{4} \nabla_{[\mu} K_{\nu]} \Gamma^{\mu \nu} \epsilon .
$$

The covariant constant spinors must be singlets under the $S U(2)$ action, and so the corresponding $L_{K} \epsilon$ must vanish. With the frames used above, this implies that $\epsilon$ is independent of the $\varphi_{j}$. The covariant constant spinors can be charged under the $U(1)$, and this charge will be correlated with helicity. When one simplifies the corresponding Lie derivative, one finds that this amounts to having:

$$
\partial_{\phi} \epsilon=i q \epsilon
$$

for some constant charge, $q$. However, by taking the flat space limit of (4.1) one can fix

$$
q=\frac{1}{2},
$$

provided that $\phi$ is normalized to the periodic range: $0 \leq \phi<2 \pi$. Finally, one can fix the normalization of $\epsilon$ by using the fact that (4.5) implies that $\bar{\epsilon} \Gamma^{\mu} \epsilon$ is constant. This means that $\epsilon$ can be chosen to be independent of $u$ and $v$.

Substituting all of this information into (4.5) yields a fairly involved system of equations that is, in fact, relatively easy to disentangle. One finds the following simple conditions:

$$
H_{5}=c_{0} H_{2}^{-1}
$$




$$
\begin{aligned}
H_{4} & =\left(\frac{1}{2} c_{0} v^{2}+c_{1}\right) H_{3}^{-1}, \\
u \partial_{u} H_{2}^{2}+c_{0} v \partial_{v} H_{6} & =0, \quad u \partial_{v} H_{1}^{2}-c_{0} v \partial_{u} H_{6}=0,
\end{aligned}
$$

where $c_{0}$ and $c_{1}$ are constants of integration. One also obtains some more complicated conditions that interrelate the derivatives of $H_{3}, H_{1}$ and $H_{2}$. The latter can be simplified and integrated by making the substitution:

$$
H_{3}^{2}=\left(\frac{1}{2} c_{0} v^{2}+c_{1}\right)\left(\frac{1+e^{2 h}}{1-e^{2 h}}\right), \quad H_{4}^{2}=\left(\frac{1}{2} c_{0} v^{2}+c_{1}\right)\left(\frac{1-e^{2 h}}{1+e^{2 h}}\right),
$$

for some function $h(u, v)$. In making this substitution we have used (4.9). One then finds that the equations for $H_{1}$ and $H_{2}$ can be integrated to give:

$$
H_{1}^{2}=\frac{c_{0} m(u)}{v\left(c_{0} v^{2}+2 c_{1}\right)} \sinh (2 h) \partial_{v} h, \quad H_{2}^{2}=-c_{0} v \partial_{v} h
$$

for some, as yet, arbitrary function $m(u)$. Using this in the remaining supersymmetry variations one finds that:

$$
H_{6}=u \partial_{u} h
$$

and thus the last equation in (4.10) yields:

$$
\frac{1}{u} \partial_{u}\left(u \partial_{u} h\right)-\frac{1}{v} \partial_{v}\left(\frac{m(u)}{v\left(c_{0} v^{2}+2 c_{1}\right)} \sinh (2 h) \partial_{v} h\right)=0 .
$$

Finally, the supersymmetry in the $\phi$-direction yields the condition

$$
\partial_{\phi} \epsilon=i\left[\frac{1}{2}+\frac{m^{\prime}(u)}{4 m(u)}\right] \epsilon,
$$

we thus find that

$$
m(u)=c_{2} u^{\alpha} \quad \Rightarrow \quad q=\left[\frac{1}{2}+\frac{\alpha}{4}\right]
$$

for some constants $c_{2}$ and $\alpha$. We thus obtain

$$
\frac{1}{u^{\alpha+1}} \partial_{u}\left(u \partial_{u} h\right)-\frac{c_{2}}{v} \partial_{v}\left(\frac{1}{v\left(c_{0} v^{2}+2 c_{1}\right)} \sinh (2 h) \partial_{v} h\right)=0 .
$$

This "master equation" determines the complete solution to our problem: Given a solution to this equation, one can determine the metric functions $H_{j}$ from (4.12), (4.11), (4.8) and (4.13). One then finds that one does indeed have a covariantly constant spinor of the form described above, and one can explicitly verify that the metric is Ricci-flat. Indeed, the form of the function $m(u)$ in (4.16) follows directly from setting the Ricci tensor to zero.

The "master equation," while non-linear, is actually a relatively simple, quasi-linear, second order PDE. It is certainly simpler than the Monge-Ampère equation, and more significantly for our work, it is very similar to the classes of PDE's found in [10, 11, [4, 12]. 


\subsection{Conical singularities and the period of $\phi$}

If we have a Ricci-flat metric of the form (3.21) then it is still, at least locally, Ricci-flat if one changes the period of $\phi$ to any value one desires. The potential cost of this is the introduction of a conical singularity, but the details will depend upon the asymptotics of $H_{1}$. If $H_{1} \rightarrow 1$ then the non-conical choice is $0 \leq \phi<2 \pi$.

The fact that one can choose the period of $\phi$ has implicitly appeared in our analysis above via the constant parameter $\alpha$. Specifically, a change of variable $u=w^{\gamma}$ combined with $\phi=\gamma \psi$ is an analytic change of variable: $z \equiv u e^{i \phi} \rightarrow z^{\gamma}$, and it conformally maps the $(u, \phi)$-metric via:

$$
d u^{2}+u^{2} d \phi^{2} \rightarrow w^{2(\gamma-1)}\left(d w^{2}+w^{2} d \psi^{2}\right) .
$$

The net result is that (3.21) keeps the same form, except that the period of $\phi$ is scaled by $\gamma$. In the foregoing analysis this change of variable merely generates a constant scale in $u \partial_{u}$, but sends $\frac{1}{u} \partial_{u}$ to $\frac{1}{\gamma w^{(2 \gamma-1)}} \partial_{w}$ which means that such a re-definition can be used to set $\alpha=0$ in (4.17). While this might be a convenient choice, one must remember that this choice imposes a particular periodicity upon the $\phi$-coordinate, and in practice one may want to preserve the free parameter $\alpha$.

\section{The complex geometry}

In order to understand the geometric significance of the solution it is instructive to re-derive them by direct analysis of the holonomy.

\subsection{The Calabi-Yau conditions}

The covariantly constant spinor defines an almost complex structure that can be expressed by choosing the hermitian vielbein

$$
\begin{aligned}
& E^{1} \equiv H_{1}(d u+i u d \phi), \\
& E^{2} \equiv H_{2} d v+i H_{5} v\left(\sigma_{3}+H_{5} d \phi\right), \\
& E^{3} \equiv H_{3} \sigma_{1}+i H_{4} \sigma_{2} .
\end{aligned}
$$

This vielbein defines the Kähler form $J=\sum_{i} E^{i} \wedge E^{\bar{\imath}}$ and a hermitian metric.

The spin connection can now be expressed in terms of holomorphic and antiholomorphic indices. In order for the spin connection to have $U(3)$ holonomy, it has 
to be a one-form with values in the Lie algebra, $\mathcal{U}(3)$. This is achieved by requiring the spin connection to be of the form $\omega^{i}{ }_{j}$ or, equivalently, $\omega_{\bar{\imath} j}$.

Since $\omega^{i}{ }_{\bar{j}} \equiv 0$, the torsion 2 -form has the following form

$$
T^{i} \equiv d E^{i}+\omega^{i}{ }_{j} \wedge E^{j}
$$

This can be decomposed into a (2,0)-form, a (1,1)-form and a $(0,2)$-form part. We will step by step require the vanishing of all these torsion parts.

First, consider the (0,2)-form part of the torsion:

$$
\left(T^{i}\right)_{(0,2)}=\left(d E^{i}\right)_{(0,2)}
$$

Requiring that this part of the torsion vanish is the same as requiring that the complex structure be integrable. For the Ansatz (5.1) the only non-trivial components of the $(0,2)$ form part of the torsion are $T^{2} \overline{1} \overline{2}, T^{3} \overline{1} \overline{3}$ and $T^{3} \overline{2} \overline{3}$. This leads to the equations

$$
\partial_{u}\left(\frac{H_{2}}{v H_{5}}\right)+\partial_{v}\left(\frac{H_{6}}{u}\right)=0, \quad \partial_{u} \log \frac{\frac{H_{3}}{H_{4}}-1}{\frac{H_{3}}{H_{4}}+1}=\frac{2 H_{6}}{u} \quad \text { and } \quad \partial_{v} \log \frac{\frac{H_{3}}{H_{4}}-1}{\frac{H_{3}}{H_{4}}+1}=-\frac{2 H_{2}}{v H_{5}}
$$

The first equation is an integrability condition and says, that there is a function $h$, such that

$$
H_{6}=u \partial_{u} h \quad \text { and } \quad \frac{H_{2}}{H_{5}}=-v \partial_{v} h
$$

The other two equations then imply, that

$$
\frac{H_{3}}{H_{4}}=-\operatorname{coth}(h+c)
$$

where $c$ is an integration constant, which can be absorbed into the definition of $h$. However, there is a second solution with $H_{3}= \pm H_{4}$, which corresponds to $c \rightarrow \pm \infty$. For now we will focus on the nondegenerate case. The Hermitean frame then reduces to

$$
\begin{aligned}
& E^{1}=H_{1}(d u+i u d \phi), \\
& E^{2}=H_{2}\left(d v-\frac{i}{\partial_{v} h}\left(\sigma_{3}+u \partial_{u} h d \phi\right)\right), \\
& E^{3}=H_{3}\left(\sigma_{1}-i \operatorname{coth}(h) \sigma_{2}\right),
\end{aligned}
$$


with the elementary $(1,0)$-forms

$$
\begin{aligned}
\omega^{1} & \equiv d u+i u d \phi \\
\omega^{2} & \equiv d v-\frac{i}{\partial_{v} h}\left(\sigma_{3}+u \partial_{u} h d \phi\right), \\
\omega^{3} & \equiv \sinh (h) \sigma_{1}-i \cosh (h) \sigma_{2} .
\end{aligned}
$$

Next, the $(1,1)$-form part of the torsion is:

$$
\left(T^{i}\right)_{(1,1)}=\left(d E^{i}\right)_{(1,1)}+\omega_{\bar{k}}^{i}{ }_{j} E^{\bar{k}} \wedge E^{j} .
$$

The vanishing of this part of the torison allows one to read off the non-trivial part of the spin connection immediately:

$$
\begin{aligned}
& \omega_{\overline{1} \overline{1} 1}=-\frac{1}{2 H_{1}} \partial_{u} \log \left(u H_{1}\right), \quad \omega_{\overline{2} \overline{1} 1}=-\frac{1}{2 H_{1}} \partial_{v} \log H_{1}, \quad \omega_{\overline{1} \overline{2} 1}=-\frac{v H_{5} \partial_{u} H_{6}}{2 u H_{1}^{2}}, \\
& \omega_{\overline{2} \overline{2} 1}=\frac{1}{4 H_{1}}\left(\partial_{u} \log \frac{H_{2}}{H_{5}}-\frac{v H_{5} \partial_{v} H_{6}}{u H_{2}}\right), \quad \omega_{\overline{1} \overline{2} 2}=-\frac{1}{4 H_{1}}\left(\partial_{u} \log \left(H_{2} H_{5}\right)+\frac{v H_{5} \partial_{v} H_{6}}{u H_{2}}\right), \\
& \omega_{\overline{2} \overline{2} 2}=-\frac{1}{2 H_{2}} \partial_{v} \log \left(v H_{5}\right), \quad \omega_{\overline{3} \overline{2} 3}=-\frac{v H_{5}}{2 H_{3} H_{4}}, \\
& \omega_{\overline{3} \overline{3} 1}=\frac{1}{4 H_{1}}\left(\partial_{u} \log \frac{H_{3}}{H_{4}}+\frac{H_{6}}{u}\left(\frac{H_{3}}{H_{4}}-\frac{H_{4}}{H_{3}}\right)\right), \\
& \omega_{\overline{3} \overline{3} 2}=\frac{1}{4 H_{2}}\left(\partial_{v} \log \frac{H_{3}}{H_{4}}-\frac{H_{2}}{v H_{5}}\left(\frac{H_{3}}{H_{4}}-\frac{H_{4}}{H_{3}}\right)\right), \\
& \omega_{\overline{1} \overline{3} 3}=-\frac{1}{4 H_{1}}\left(\partial_{u} \log \left(H_{3} H_{4}\right)+\frac{H_{6}}{u}\left(\frac{H_{3}}{H_{4}}+\frac{H_{4}}{H_{3}}\right)\right), \\
& \omega_{\overline{2} \overline{3} 3}=\frac{1}{4 H_{2}}\left(-\partial_{v} \log \left(H_{3} H_{4}\right)+\frac{H_{2}}{v H_{5}}\left(\frac{H_{3}}{H_{4}}+\frac{H_{4}}{H_{3}}\right)\right) .
\end{aligned}
$$

One can use the reality relation, $\omega_{k \bar{\imath} j}=-\left(\omega_{\bar{k} \bar{j} i}\right)^{*}$, to determine the remainder of the spin connection.

The the spin connection can now be inserted into the equation for the vanishing of the $(2,0)$-form part of the torsion. This is equivalent to the Kähler condition $d J=0$, and reduces to four non-trivial equations:

$$
\begin{aligned}
& \partial_{v}\left(H_{1}^{2}\right)=\frac{v}{u} H_{2} H_{5} \partial_{u} H_{6}, \\
& \partial_{u}\left(H_{2} H_{5}\right)=0, \quad \partial_{v}\left(H_{3} H_{4}\right)=v H_{2} H_{5} \quad \text { and } \quad \partial_{u}\left(H_{3} H_{4}\right)=0 .
\end{aligned}
$$


The last three equations show that $H_{3} H_{4}$ can be an arbitrary function, $f(v)$, and that $H_{2} H_{5}$ is given by $H_{2} H_{5}=\frac{1}{v} \partial_{v} f(v)$. Fixing $f(v)$ fixes the freedom to re-define $v$ by an arbitrary function of $\tilde{v}(v)$. Using (5.5) and (5.6), we get the relations

$$
H_{3}^{2}=f(v) \operatorname{coth}(h) \quad \text { and } \quad H_{2}^{2}=\partial_{v} h \partial_{v} f .
$$

The first condition (5.11) then determines $H_{1}^{2}$ up to a function of $u$ only.

Finally, we have the condition for $S U(3)$ holonomy, as opposed to $U(3)$ holonomy. From the spin connection we can extract a $U(1)$ connection $\tilde{\omega}=\omega^{i}{ }_{i}$. Requiring that $\tilde{\omega}$ is a flat connection is now equivalent to $\omega$ having $S U(3)$ holonomy. This condition is $d \tilde{\omega}=0$ or $\tilde{\omega}=d \lambda$. Using (5.10), we see that

$$
\tilde{\omega}=\frac{i u}{2} \partial_{u} \log \frac{u^{2} H_{1}^{2} H_{2}^{2} H_{3}^{2}}{\left(\partial_{v} h\right)^{2} \cosh ^{2} h} d \phi-\frac{i}{2 \partial_{v} h} \partial_{v} \log \frac{u^{2} H_{1}^{2} H_{2}^{2} H_{3}^{2}}{\left(\partial_{v} h\right)^{2} \cosh ^{2} h}\left(\sigma_{3}+u \partial_{u} h d \phi\right) .
$$

A way to make this a total derivative is by requiring, that

$$
\frac{u^{2} H_{1}^{2} H_{2}^{2} H_{3}^{2}}{\left(\partial_{v} h\right)^{2} \cosh ^{2} h}=c_{2} u^{\alpha+2} .
$$

Inserting this together with (5.12) into the first equation of (5.11) gives the master equation

$$
\frac{c_{2}}{\partial_{v} f} \partial_{v}\left(\frac{1}{\partial_{v}\left(f^{2}\right)} \sinh (2 h) \partial_{v} h\right)+\frac{1}{u^{\alpha+1}} \partial_{u}\left(u \partial_{u} h\right)=0,
$$

where $c_{2}$ can be absorbed into $f$. Choosing $f$ properly, one can easily recover (4.17).

The Calabi-Yau condition can also be formulated in terms of a holomorphic $(3,0)$-form

$$
\Omega \equiv e^{i \phi} E^{1} \wedge E^{2} \wedge E^{3},
$$

where the phase dependence can be fixed by requiring the proper transformation properties under the $\mathcal{R}$-symmetry. To have a Calabi-Yau manifold this form must be holomorphic, and satisfy

$$
\Omega \wedge \bar{\Omega}=\frac{1}{3 !} J \wedge J \wedge J .
$$

The latter is trivially satisfied, while the former is equivalent to requiring that $\Omega$ be closed. This leads again to (5.15).

In general it is easy in the non-coordinate base to impose (5.17). But then it is harder to impose that $J$ and $\Omega$ are closed. On the other hand in a coordinate base it is easy to impose that $J$ and $\Omega$ are closed, but then it is hard to impose (5.17). The true art is to impose both at the same time. 


\subsection{Holomorphic coordinates}

Finally, it is instructive to see how the conifold is parametrized in terms of the new variables, $u$ and $v$. To determine the relationship one notes that (3.14) and (5.8) must be linear combinations of one another. It is trivial to solve this system and thereby obtain:

$$
\mu=u \cosh (h), \quad \nu=u \sinh (h) .
$$

One can then compute the holomorphic coordinates explicitly using (3.8) and (3.9) to obtain:

$$
\begin{aligned}
& w_{1}=\frac{1}{2}\left(i w_{4}\right)\left[\cos ^{2}\left(\frac{1}{2} \varphi_{1}\right)\left(\xi e^{i \varphi_{2}}+\left(\xi e^{i \varphi_{2}}\right)^{-1}\right)-\sin ^{2}\left(\frac{1}{2} \varphi_{1}\right)\left(\xi e^{-i \varphi_{2}}+\left(\xi e^{-i \varphi_{2}}\right)^{-1}\right)\right] \\
& w_{2}=\frac{i}{2}\left(i w_{4}\right)\left[\cos ^{2}\left(\frac{1}{2} \varphi_{1}\right)\left(\xi e^{i \varphi_{2}}-\left(\xi e^{i \varphi_{2}}\right)^{-1}\right)+\sin ^{2}\left(\frac{1}{2} \varphi_{1}\right)\left(\xi e^{-i \varphi_{2}}-\left(\xi e^{-i \varphi_{2}}\right)^{-1}\right)\right],
\end{aligned}
$$

and

$$
w_{3}=\frac{1}{2}\left(i w_{4}\right)\left(\xi+\xi^{-1}\right) \sin \varphi_{1}, \quad w_{4}=-i u e^{i \phi}
$$

where

$$
\xi \equiv e^{h+i \varphi_{3}}
$$

One can naturally parametrize $\mathbb{C}^{2}$ using the $S U(2)$ matrix $\mathcal{L}$ and a radial coordinate, $r$, and one can obtain:

$$
\zeta_{1}=r \cos \left(\frac{1}{2} \varphi_{1}\right) e^{\frac{i}{2}\left(\varphi_{2}+\varphi_{3}\right)}, \quad \zeta_{2}=i r \sin \left(\frac{1}{2} \varphi_{1}\right) e^{-\frac{i}{2}\left(\varphi_{2}-\varphi_{3}\right)}
$$

Taking $r=e^{h / 2}$, we see that (5.19) and (5.20) may be rewritten as:

$$
\begin{aligned}
& w_{1}=\frac{1}{2}\left(i w_{4}\right)\left[\left(\zeta_{1}^{2}+\zeta_{2}^{2}\right)+e^{-2 h}\left(\bar{\zeta}_{1}^{2}+\bar{\zeta}_{2}^{2}\right)\right] \\
& w_{2}=\frac{i}{2}\left(i w_{4}\right)\left[\left(\zeta_{1}^{2}-\zeta_{2}^{2}\right)-e^{-2 h}\left(\bar{\zeta}_{1}^{2}-\bar{\zeta}_{2}^{2}\right)\right] \\
& w_{3}=-\frac{i}{2}\left(i w_{4}\right)\left(\zeta_{1} \zeta_{2}+e^{-2 h} \bar{\zeta}_{1} \bar{\zeta}_{2}\right), \quad w_{4}=-i u e^{i \phi} .
\end{aligned}
$$

This shows how we are writing the conifold as a deformation of the orbifold (3.7), and that the confold and orbifold have distinct complex structures.

Alternatively, one can take:

$$
\zeta_{1}=r \cos \left(\frac{1}{2} \varphi_{1}\right) e^{-\frac{i}{2}\left(\varphi_{3}+\varphi_{2}\right)}, \quad \zeta_{2}=i r \sin \left(\frac{1}{2} \varphi_{1}\right) e^{-\frac{i}{2}\left(\varphi_{3}-\varphi_{2}\right)}
$$


and set $r=e^{-h / 2}$. One then finds that (5.23) and (5.20) may be rewritten as:

$$
\begin{aligned}
& w_{1}=\frac{1}{2}\left(i w_{4}\right)\left[\left(\zeta_{1}^{2}+\zeta_{2}^{2}\right)+e^{2 h}\left(\bar{\zeta}_{1}^{2}+\bar{\zeta}_{2}^{2}\right)\right], \\
& w_{2}=-\frac{i}{2}\left(i w_{4}\right)\left[\left(\zeta_{1}^{2}-\zeta_{2}^{2}\right)-e^{2 h}\left(\bar{\zeta}_{1}^{2}-\bar{\zeta}_{2}^{2}\right)\right], \\
& w_{3}=-\frac{i}{2}\left(i w_{4}\right)\left(\zeta_{1} \zeta_{2}+e^{2 h} \bar{\zeta}_{1} \bar{\zeta}_{2}\right), \quad w_{4}=-i u e^{i \phi} .
\end{aligned}
$$

One of the important lessons in this coordinate change exercise is that the master function, $h$, appears as a natural coordinate on the conifold, and one may view our approach to finding the Ricci-flat metric as prescribing the metric functions and then solving equations to determine the complex coordinates. The simplification that the "master equation" provides over the Monge-Ampère equation may thus be viewed as a clever re-definition of dependent and independent variables.

\subsection{The conifold again}

It is relatively easy to obtain the conifold solution from our re-formulation of the problem of finding Ricci-flat metrics: One simply sets (3.21) equal to (3.17) and extracts the necessary re-definitions. The easiest equations follow from angular parts of the metric and one immediately finds that:

$$
h=\log \left[\tan \left(\frac{\theta}{2}\right)\right]
$$

along with

$$
v^{2}=\frac{2}{3 c_{0}} \rho^{2} \cos \theta
$$

and $c_{1}=0$. Given this, it is then easy to find the other change of variable:

$$
u^{4}=\frac{4}{81 c_{2} c_{0}^{2}} \rho^{6} \sin ^{4} \theta
$$

and show that the metrics match perfectly for any value of $c_{0}$ and $c_{2}$ if and only if $\alpha=2$ in (4.16). Thus the master function (5.26) satisfies:

$$
\frac{1}{u^{3}} \partial_{u}\left(u \partial_{u} h\right)-\frac{c_{2}}{c_{0} v} \partial_{v}\left(\frac{1}{v^{3}} \sinh (2 h) \partial_{v} h\right)=0 .
$$

Since the dependence on $c_{0}$ and $c_{2}$ in (5.27)-(5.29) can be removed by rescaling $u$ and $v$, we can simply set $c_{0}=c_{2}=1$.

One can also derive this result $a b$ initio. That is, one can seek a metric that is a cone over a five-dimensional Einstein space. that depends upon a single variable $\theta$. Denote the 
radial coordinate on the cone by $\rho$, then by taking ratios of metric coefficients one sees from (3.21) that $h$ can only be a function of $\theta$, and so $v=\rho q_{2}(\theta)$ for some function $q_{2}$ provided $c_{1}=0$. It thus follows that $H_{5}$ and hence $H_{2}$ will be independent of $\rho$. Then, from the $d \phi^{2}$ terms we see that $H_{1} u=\rho q_{3}(\theta)$, for some function, $q_{3}$. Using this in the expression for $H_{1}^{2} / H_{2}^{2}$ that one obtains from (4.12) one finds:

$$
u=\rho^{\frac{6}{\alpha+2}} q_{1}(\theta), \quad v=\rho q_{2}(\theta) .
$$

In particular, one finds that

$$
x \equiv \frac{u^{\frac{1}{2}(\alpha+2)}}{v^{3}}
$$

is independent of $\rho$, and is only a function of $\theta$. One can thus take $h=h(x)$, and substitute into the master equation. For $\alpha=2$ and $c_{0}=c_{2}=1$, this yields:

$$
\partial_{x}\left(x \partial_{x} h\right)=\frac{3}{8} x^{2}\left(7 \partial_{x}(\cosh (2 h))+3 x \partial_{x}^{2}(\cosh (2 h))\right)
$$

\subsection{Monge-Ampère - Tian-Yau}

The master equation (4.17) is a quasilinear PDE, but it is very hard to solve analytically. However, Tian and Yau [23,24] proved some existence and uniqueness theorems for non-compact Calabi-Yau metrics. Here we will remark upon how one might employ their methods to prove that there is a solution to the master equation with the desired properties.

One can construct a closed Kähler form, $J_{0}$, with the right asymptotics on the noncompact Calabi-Yau manifold. This Kähler form defines the Kähler class of the desired solution. Since the manifold is Calabi-Yau, there exists a holomorphic (3,0)-form $\Omega$. This 3 -form is not unique, since the space is noncompact, but we can fix $\Omega$ by requiring the right asymptotics and symmetry properties.

A Ricci-flat, Kähler metric has the property

$$
\frac{1}{3 !} J \wedge J \wedge J=\Omega \wedge \bar{\Omega}
$$

The two-form, $J_{0}$, typically does not satisfy this equation. However one can try to find a potential $\mathcal{K}$, such that

$$
\frac{1}{3 !}\left(J_{0}+\partial \bar{\partial} \mathcal{K}\right) \wedge\left(J_{0}+\partial \bar{\partial} \mathcal{K}\right) \wedge\left(J_{0}+\partial \bar{\partial} \mathcal{K}\right)=\Omega \wedge \bar{\Omega}
$$


This equation is a Monge-Ampère equation, which is much more difficult than our master equation. However, Tian and Yau show, that there is a unique potential $\mathcal{K}$ with the right fall-off properties at infinity. The proof of this theorem respects all the symmetries of the problem, but it does not allow for a singularity at a finite point. There should however be a simple generalization of the proof, where the asymptotics of the Kähler form at the singularity is specified.

We will now argue, that there is a $J_{0}$ with the right asymptotics at the singularity and at infinity. First, there is the singular conifold metric. This metric has the right asymptotics at the singularity. On the other hand there is the degenerate limit of the conifold, which is $\mathbb{C}^{2} / \mathbb{Z}_{2} \times \mathbb{C}$. One can now use a bump function to glue the two Kähler forms to a single Kähler form with the right asymptotics. The modification of the TianYau theorem should then prove the existence and uniqueness of a Ricci flat Kähler metric with the desired asymptotics on the singular conifold.

\subsection{Completing the solution}

We have been implicitly using the results of [25,20] that imply that the internal metric we seek must be a Calabi-Yau geometry. The complete solution must involve non-trivial, harmonic warp factors and related five-form fluxes as in (2.15) and (2.16). This, in principle, requires us to solve the Laplace equation for the metric described above. Amusingly, this equation, while linear, is not greatly different from the master equation itself. However, without knowing the function, $h$, we do not appear to be able to find the requisite solution analytically. On the other hand, we know that we need an $O(3) \times U(1)$ symmetric, smooth harmonic function on this space with the appropriate asymptotics at the singularity and at infinity. Again, there should be a unique such function.

All of this strongly suggests that the Klebanov-Witten flow is actually captured by our master equation.

\section{The geometry of the PW solution}

There is a striking similarity between the analysis above and the analysis of more general families of flows that involve fluxes [10, 11, [1, 12]. Moreover the master equation derived above is very similar to the equations that govern these more general flows. Given this, we now wish to revisit one of these solutions to elucidate the underlying geometry in the light of what we have seen above. 
The flow we consider is, of course, the close cousin of the Klebanov-Witten flow in which the mass perturbation is of the form (2.2) but with both terms having the same sign. This flow lies purely within the untwisted sector of the gauge theory, and its holographic dual involves non-trivial three-form fluxes. The $S U(2) \times U(1)$ invariant families of flow solutions of this type were analyzed in [7].

\subsection{The elements of the PW solution}

We begin by recalling some of the details of the solution of [7], but we use slightly modified conventions. We start by recasting the metric Ansatz in the form:

$$
d s^{2}=H_{0}^{2}\left(\eta_{\mu \nu} d x^{\mu} d x^{\nu}\right)-H_{0}^{-2} d s_{6}^{2},
$$

where $d s_{6}^{2}$ is given by (3.21) with $H_{3}=H_{4}=\frac{1}{2} v$. Note the inclusion of the factor of $H_{0}^{-2}$ in front of $d s_{6}^{2}$. We thus use the frames:

$$
\begin{aligned}
& e^{a}=H_{0} d x^{a}, \quad a=1, \ldots, 4, \quad e^{5}=H_{0}^{-1} H_{1} d u, \quad e^{6}=H_{0}^{-1} H_{2} d v, \\
& e^{7}=\frac{1}{2} H_{0}^{-1} v \sigma_{1}, \quad e^{8}=\frac{1}{2} H_{0}^{-1} v \sigma_{2}, \\
& e^{9}=\frac{1}{2} H_{0}^{-1} H_{5} v\left(\sigma_{3}+H_{6} d \phi\right), \quad e^{10}=H_{0}^{-1} H_{1} u d \phi,
\end{aligned}
$$

Following [7], we define:

$$
\Psi=\log \left(v^{2} \frac{H_{1}}{H_{2}}\right) .
$$

Then the metric coefficients of $d s_{6}^{2}$ are determined by:

$$
H_{1}^{2}=\frac{1}{2 v^{3}} e^{2 \Psi} \partial_{v} \Psi, \quad H_{2}^{2}=\frac{1}{2} v \partial_{v} \Psi, \quad H_{5}=H_{2}^{-1}, \quad H_{6}=u \partial_{u} \Psi,
$$

\subsection{The complex geometry of the $P W$ solution}

Observe that, with the exception of the expression for $H_{1}$, the PW solution is identical to the form of the metric discovered in sections 4 and 5. Indeed, if one takes:

$$
h=\Psi+k,
$$

and takes the limit $k \rightarrow \infty$ while scaling $c_{2}$ appropriately, then the metric of section 4 matches the form of the metric above. 
Thus, the metric in [7] is hermitian with an integrable complex structure, with $(1,0)$ forms

$$
\omega_{1}=d u-i u d \phi, \quad \omega_{2}=\sigma_{1}+i \sigma_{2}, \quad \omega_{3}=d \Psi+i \sigma_{3} .
$$

The corresponding holomorphic coordinates are:

$$
z_{1}=u e^{-i \phi}, \quad z_{2}=e^{\frac{1}{2} \Psi} \cos \left(\frac{1}{2} \varphi_{1}\right) e^{\frac{i}{2}\left(\varphi_{2}+\varphi_{3}\right)}, \quad z_{3}=e^{\frac{1}{2} \Psi} \sin \left(\frac{1}{2} \varphi_{1}\right) e^{-\frac{i}{2}\left(\varphi_{2}-\varphi_{3}\right)}
$$

The putative almost complex structure:

$$
J=-H_{1}^{2} u d u \wedge d \phi+\frac{1}{4} v^{2} \sigma_{1} \wedge \sigma_{2}+\frac{1}{2} v d v \wedge\left(\sigma_{3}+u \partial_{u} \Psi\right)
$$

is thus a true complex structure, and the underlying complex manifold is simply $\mathbb{C}^{2} / \mathbb{Z}_{2} \times \mathbb{C}$ parametrized by (6.7).

Moreover, the (3,0)-form:

$$
\Omega=e^{\Psi-i \phi} \omega_{1} \wedge \omega_{2} \wedge \omega_{3}=-\frac{1}{4} d z_{1} \wedge d z_{2} \wedge d z_{3}
$$

is manifestly closed, and thus holomorphic. It also satisfies the Calabi-Yau condition:

$$
\Omega \wedge \bar{\Omega}=\frac{64}{3} i J \wedge J \wedge J
$$

and thus there is a holomorphic $(3,0)$ form that is a square root of the volume form. We thus have a Calabi-Yau manifold, except that the manifold is not Kähler. The complex structure (or, more correctly, the Kähler form) is not closed.

The solution of [7] requires that $\Psi$ satisfy:

$$
u^{3} \frac{\partial}{\partial u}\left(\frac{1}{u^{3}} \frac{\partial}{\partial u} \Psi\right)+\frac{1}{v} \frac{\partial}{\partial v}\left(\frac{1}{v^{3}} e^{2 \Psi} \frac{\partial \Psi}{\partial v}\right)=0,
$$

whereas the Kähler condition, or Ricci-flatness of the metric, would require

$$
\frac{1}{u} \frac{\partial}{\partial u}\left(u \frac{\partial}{\partial u} \Psi\right)+\frac{1}{v} \frac{\partial}{\partial v}\left(\frac{1}{v^{3}} e^{2 \Psi} \frac{\partial \Psi}{\partial v}\right)=0 .
$$

Thus the solution of [7] is based upon a the complex manifold $\mathbb{C}^{3}$, endowed with hermitian, non-Kähler metric. The mysterious master equation of [7] thus turns out to be simple deformation of the Kähler condition.

It is also worth noting that if one sets $h= \pm(\Psi+k)$, where $k$ is a constant, in (4.17) and takes the limit $k \rightarrow \infty$ with $c_{2} \rightarrow 0$ then (4.17) (for $\alpha=2$ and $c_{1}=0$ ) degenerates to (6.12). This substitution of $h= \pm(\Psi+k)$ and limit of $k$ is precisely the limit that degenerates the conifold to the orbifold, and so it is to be expected that the "master equation" degenerate to the Ricci-flat condition for $\mathbb{C}^{2} / \mathbb{Z}_{2} \times \mathbb{C}$, and hence equation (6.12). 


\section{Conclusions}

Our purpose has been to elucidate the geometry that underlies the holographic duals of $\mathcal{N}=1$ supersymmetric flows from $\mathcal{N}=2$ quiver gauge theories. We are particularly interested in flows generated by mass terms for the chiral matter multiplets in the $\mathcal{N}=$ 2 vector multiplets. The twisted sector masses are dual to blow-up modes in the dual geometry while the 'total' mass parameter comes from the untwisted sector and is dual to

a three-from flux. Here, for simplicity, we have focussed on the $\widehat{A}_{1}$ quiver theory, but we believe that our results apply in general. Moreover, we studied the two extreme cases: a flow purely in the twisted sector (the Klebanov-Witten flow) and the PW flow.

We have argued that flows purely in the twisted sector must have an underlying CalabiYau geometry all along the flow, and that the complete solution is simply an extension via the harmonic Ansatz. For the $\widehat{A}_{1}$ quiver we showed that this means that the underlying manifold must be the singular conifold with a Calabi-Yau metric that interpolates between the orbifold point at infinity and the cone over $T^{(1,1)}$ in the deep interior. The standard approach to finding such a metric would involve the solving the Monge-Ampère equation, a sixth order, non-linear PDE. However, by using the symmetries and structure of the flow we were able to make an Ansatz of sufficient generality to capture the solution and yet reduce everything to the solution of a single, second order quasi-linear PDE. This substantial simplification compared to the Monge-Ampère approach arises partially because we have a higher level of symmetry, and do not need the full force of the general method. The other reason for the simplification is because of an inversion of dependent and independent variables: Monge-Ampère approach leads to differential equations for metric functions in terms of fixed complex coordinates, while the "master equation" is really a differential equation for part of the complex coordinates having fixed some of the metric functions. At any rate, the "master equation" is, on the face of it, far simpler than the Monge-Ampère equation, and the master equation has a very simple linearization that leads to an obvious perturbation expansion.

One of the other consequences of this description of a Calabi-Yau manifold by the master equation (6.12) is that it sheds some light upon other non-Calabi-Yau, flux solutions, like those of [10, [1], [1, [12], that are completely determined by a very, very similar such equation. This strongly suggest that such solutions must be relatively simple modifications of the Calabi-Yau condition. To understand this better, we re-examined the geometry underlying the solution of [7], and showed that this solution is essentially a a non-Kähler 
Calabi-Yau manifold: It has an integrable complex structure, a hermitian metric, and a holomorphic $(3,0)$ form that is the square root of the volume form. We are continuing the study of this geometry and its generalizations, and we would particularly like to understand the geometric meaning of the simple deformations of the master equations that take one from the Calabi-Yau solutions to the non-trivial flux solutions.

It is also useful to recall that the master equation, (6.11) possesses a known, highly non-trivial solution coming from gauged supergravity [ descent flow on a simple five-dimensional superpotential that provides one of the most interesting flows to the non-trivial IR fixed point, and while this solution is not given analytically, its solution is trivially obtained graphically. If one could forge the connection between the various master equations more deeply, one might hope to find special CalabiYau solutions by such simple, graphical techniques.

There is also the work of [8,9] concerning the continuous family of flows involving arbitrary mixtures of mass terms in the twisted and untwisted sectors. Moreover, it was argued in [8] that all these flows should have a continuous family if IR fixed points. We

are presently generalizing the results presented here so as to try to capture this broader family of flows.

We hope, ultimately, to be able to give a complete geometric characterization of supersymmetric backgrounds involving fluxes. It is evident from the results presented here and elsewhere that trying to characterize the supersymmetry bundle directly is a very effective approach in studying not only these problems, but also in re-visiting Calabi-Yau geometries.

\section{Acknowledgements}

This work was supported in part by funds provided by the U.S. Department of Energy under grant number DE-FG03-84ER-40168. In addition, N.H. has been supported by the Fletcher-Jones graduate fellowship at USC, while K.P. has been supported in part by the grant \# SAB2002-0001 from the Ministerio de Educación, Cultura y Deporte of Spain. K.P. would like to thank the members of the Departament ECM for their hospitality during his sabbatical at the University of Barcelona. We would also like to thank R. Corrado for helpful conversations. 


\section{References}

[1] I. R. Klebanov and E. Witten, "Superconformal field theory on threebranes at a Calabi-Yau singularity," Nucl. Phys. B 536, 199 (1998) arXiv:hep-th/9807080].

[2] S. Gubser, N. Nekrasov and S. Shatashvili, "Generalized conifolds and four dimensional N = 1 superconformal theories," JHEP 9905, 003 (1999) arXiv:hepth/9811230|.

[3] A. Khavaev, K. Pilch and N. P. Warner, "New vacua of gauged N = 8 supergravity in five dimensions," Phys. Lett. B 487, 14 (2000) arXiv:hep-th/9812035.

[4] D. Z. Freedman, S. S. Gubser, K. Pilch and N. P. Warner, "Renormalization group flows from holography supersymmetry and a c-theorem," Adv. Theor. Math. Phys. 3, 363 (1999) arXiv:hep-th/9904017.

[5] K. Pilch and N. P. Warner, "A new supersymmetric compactification of chiral IIB supergravity," Phys. Lett. B 487, 22 (2000) arXiv:hep-th/0002192.

[6] K. Pilch and N. P. Warner, " $\mathrm{N}=1$ supersymmetric renormalization group flows from IIB supergravity," Adv. Theor. Math. Phys. 4, 627 (2002) arXiv:hep-th/0006066.

[7] K. Pilch and N. P. Warner, "N = 1 supersymmetric solutions of IIB supergravity from Killing spinors," arXiv:hep-th/0403005.

[8] R. Corrado, M. Gunaydin, N. P. Warner and M. Zagermann, "Orbifolds and flows from gauged supergravity," Phys. Rev. D 65, 125024 (2002) [arXiv:hep-th/0203057].

[9] R. Corrado and N. Halmagyi, "N = 1 field theories and fluxes in IIB string theory," arXiv:hep-th/0401141.

[10] C. N. Gowdigere, D. Nemeschansky and N. P. Warner, "Supersymmetric solutions with fluxes from algebraic Killing spinors," arXiv:hep-th/0306097.

[11] K. Pilch and N. P. Warner, "Generalizing the $\mathrm{N}=2$ supersymmetric RG flow solution of IIB supergravity," Nucl. Phys. B 675, 99 (2003) arXiv:hep-th/0306098.

[12] D. Nemeschansky and N. P. Warner, "A family of M-theory flows with four supersymmetries," arXiv:hep-th/0403006.

[13] C. Gowdigere, D. Nemeschansky, K. Pilch, and N.P. Warner, to appear.

[14] R. G. Leigh and M. J. Strassler, "Exactly marginal operators and duality in fourdimensional N=1 supersymmetric gauge theory," Nucl. Phys. B 447, 95 (1995) arXiv:hep-th/9503121.

[15] M. R. Douglas and B. R. Greene, "Metrics on D-brane orbifolds," Adv. Theor. Math. Phys. 1, 184 (1998) arXiv:hep-th/9707214.

[16] M. R. Douglas and G. W. Moore, "D-branes, Quivers, and ALE Instantons," arXiv:hep-th/9603167.

[17] C. V. Johnson, K. J. Lovis and D. C. Page, "The Kähler structure of supersymmetric holographic RG flows," JHEP 0110, 014 (2001) arXiv:hep-th/0107261. 
[18] K. Becker, M. Becker and A. Strominger, "Five-branes, membranes and nonperturbative string theory," Nucl. Phys. B 456, 130 (1995) [arXiv:hep-th/9507158].

[19] M. Berkooz, M. R. Douglas and R. G. Leigh, "Branes intersecting at angles," Nucl. Phys. B 480, 265 (1996) arXiv:hep-th/9606139.

[20] M. Grana and J. Polchinski, "Gauge / gravity duals with holomorphic dilaton," Phys. Rev. D 65, 126005 (2002) arXiv:hep-th/0106014.

[21] P. Candelas and X. C. de la Ossa, "Comments On Conifolds," Nucl. Phys. B 342, 246 (1990).

[22] L. J. Romans, "New Compactifications Of Chiral N=2 D = 10 Supergravity," Phys. Lett. B 153, 392 (1985).

[23] G. Tian and S. T. Yau, "Complete Kähler Manifolds with Zero Ricci Curvature. I," Journal of the American Mathematical Society, vol. 3, number 3, July 1990, p. 579

[24] G. Tian and S. T. Yau, "Complete Kähler Manifolds with Zero Ricci Curvature. II," Invent. Math. 106, 27-60 (1991)

[25] K. Becker and M. Becker, "M-Theory on Eight-Manifolds," Nucl. Phys. B 477, 155 (1996) arXiv:hep-th/9605053. 